\title{
Testing electric field models using ring current ion energy spectra from the Equator-S ion composition (ESIC) instrument
}

\author{
L. M. Kistler ${ }^{1}$, B. Klecker ${ }^{2}$, V. K. Jordanova ${ }^{1}$, E. Möbius ${ }^{1}$, M. A. Popecki ${ }^{1}$, D. Patel ${ }^{1}$, J. A. Sauvaud ${ }^{3}$, H. Rème ${ }^{3}$, \\ A. M. Di Lellis ${ }^{4}$, A. Korth ${ }^{5}$, M. McCarthy ${ }^{6}$, R. Cerulli ${ }^{4}$, M. B. Bavassano Cattaneo ${ }^{4}$, L. Eliasson ${ }^{7}$, \\ C. W. Carlson ${ }^{8}$, G. K. Parks ${ }^{6}$, G. Paschmann ${ }^{2}$, W. Baumjohann ${ }^{2}$, G. Haerendel ${ }^{2}$ \\ ${ }^{1}$ Space Science Center, Morse Hall, University of New Hampshire, Durham, NH, USA \\ E-mail: Lynn.kistler@uhn.edu \\ ${ }^{2}$ Max-Planck-Institut für Extraterrestriche Physik, Garching, Germany \\ ${ }^{3}$ C.E.S.R.., Toulouse, France \\ ${ }^{4}$ I.F.S.I, Rome, Italy \\ ${ }^{5}$ Max-Planck-Institut für Aeronomie, Katlinberg-Lindau, Germany \\ ${ }^{6}$ University of Washington, Seattle, WA, USA \\ ${ }^{7}$ Swedish Institute of Space Physics, Kiruna, Sweden \\ ${ }^{8}$ University of California, Berkeley, CA, USA
}

Received: 26 March 1999 / Revised: 9 August 1999 / Accepted: 17 August 1999

\begin{abstract}
During the main and early recovery phase of a geomagnetic storm on February 18, 1998, the Equator-S ion composition instrument (ESIC) observed spectral features which typically represent the differences in loss along the drift path in the energy range $(5-15 \mathrm{keV} / \mathrm{e})$ where the drift changes from being $\mathbf{E} \times \mathbf{B}$ dominated to being gradient and curvature drift dominated. We compare the expected energy spectra modeled using a Volland-Stern electric field and a Weimer electric field, assuming charge exchange along the drift path, with the observed energy spectra for $\mathrm{H}^{+}$ and $\mathrm{O}^{+}$. We find that using the Weimer electric field gives much better agreement with the spectral features, and with the observed losses. Neither model, however, accurately predicts the energies of the observed minima.
\end{abstract}

Key words. Magnetospheric physics (energetic particles trapped; plasma convection; storms and substorms)

\section{Introduction}

During geomagnetic storms, the ion energy spectra in the ring current show minima which reflect the drift history of the ion population. Over the energy range from 1 to $50 \mathrm{keV} / \mathrm{e}$, the dominant drift changes from the eastward and sunward $\mathbf{E} \times \mathbf{B}$ drift at low energies, to the westward gradient and curvature drifts at higher energies. In the energy range where the two contributions are approximately equal, the drifts are very

Correspondence to: L. M. Kistler sensitive to the exact electric and magnetic field configurations. The ion spectra observed on the dayside typically contain one or more minima which correspond to the drift paths on which the greatest losses occur, either because they have the longest drift time, or because they drift the closest to the Earth, where losses are greater. Many studies (e.g., Kistler et al., 1989; Fok et al., 1996; Jordanova et al., 1999) have shown qualitative agreement between observed and predicted spectra based on these principles. However, there has been difficulty in getting quantitative agreement. The reason for this is most likely that the electric and magnetic fields used for modeling the spectra have been simplified.

The most common electric field used for this type of modeling is the Volland-Stern (Volland, 1973; Stern, 1975) electric field. The Volland-Stern field is derived from the potential:

$U_{\text {conv }}=A R_{o}^{\gamma} \sin (\varphi)$

where $R_{o}$ is the radial distance in the equatorial plane, $\gamma$ is an adjustable shielding parameter, normally taken to be $2, \varphi$ is the azimuthal angle, measured eastward from midnight, and $A$ gives the magnitude. Maynard and Chen (1975) determined an empirical formula for $A$ as a function of the planetary index $K p$, for the $\gamma=2$ case. This field has the advantage of being analytically very simple, and therefore easy to use in numerical models. However, recent measurements indicate that it may be very far off from the actual electric field, particularly during storm times (Wygant et al., 1998). A more recent model with a much stronger empirical basis is the Weimer model (Weimer, 1995, 1996). This model is based on measurements of the electric potential by the vector electric field instrument on the DE2 spacecraft. The data were sorted into groups by IMF clock angle, IMF magnitude and dipole tilt angle, and then the 
measurements from each group were used to derive a model in terms of a spherical harmonic expansion. By using the measured IMF parameters, this model can be used to give a time dependent estimate of the electric field. The model gives the potential at low altitudes. This potential must then be mapped up the field line to get the equatorial electric field.

In this work, we show one example of a geomagnetic storm where large flux enhancements were observed in the ring current. The energy spectra as observed with the Equator-S ion composition (ESIC) instrument showed the features that result from losses along drift paths. We will use both the Volland-Stern and the Weimer electric fields combined with a dipole magnetic field to determine the drift trajectories of ions during a geomagnetic storm. We will then determine the expected energy spectra on the dayside, assuming that charge exchange is the only loss process, and compare the expected energy spectra with those observed.

\section{Instrumentation}

The Equator-S satellite is in a highly elliptical orbit, with an apogee of $11.3 \mathrm{Re}$ and a perigee of $500 \mathrm{~km}$. It orbits in the geographic equatorial plane. The initial apogee was at 11:00 LT, and it then precesses toward earlier local times. The data shown here are mainly from the Equator-S ion composition instrument (ESIC). ESIC measures the 3-dimensional distribution functions of the major ion species in the magnetosphere and magnetosheath over the energy per charge range $20-40000 \mathrm{eV} / \mathrm{e}$. It is a combination of a top-hat electrostatic analyzer followed by post-acceleration by $15-18 \mathrm{kV}$ and a timeof-flight measurement. It is similar to the CODIF (CIS1) instrument designed for CLUSTER (Rème et al., 1997) and the TEAMS instrument on FAST (Möbius et al., 1998). It can resolve the major ion species, $\mathrm{H}^{+}, \mathrm{He}^{++}$, $\mathrm{He}^{+}$and $\mathrm{O}^{+}$.

The electrostatic analyzer is divided into two halves, with geometric factors different by a factor of 100 . Only one half operates at a time, giving a $180^{\circ}$ instantaneous field of view divided into eight sectors of $22.5^{\circ}$ each. The full 3D distribution is achieved using the spin. The electrostatic analyzer sweeps through the full energy range 16 times per spin, so that the full distribution is obtained in one spin.

An on-board processor collects the event data from the sensor and classifies each event by mass, energy, and angle. It then bins the data and creates "data products" which consist of 3-dimensional (3D) distributions, a mass spectrum, and moments of the distribution. In addition products containing the raw count rates from the sensor and a sample of raw data from individual ions, including the time-of-flight, position, spin sector, and energy step are also generated to monitor the performance and calibration of the sensor. These 3D distribution products are available for each of the four major species, $\mathrm{H}^{+}, \mathrm{O}^{+}, \mathrm{He}^{++}$, and $\mathrm{He}^{+}$, with either 16 or 32 energy bins, and 88,24 , or 12 angular bins. For the mass spectrum, the mass space from 0 to $70 \mathrm{AMU}$ is divided into 31 bins spaced as the square-root of the mass, with a factor of four discontinuity at 9 AMU. This product allows minor species such as $\mathrm{O}^{++}$to be detected, and is also useful for determining the rate of background in the instrument, as will be illustrated. The combination of products obtained at any time and their time resolution depends on the telemetry rate and the expected count rates for the particular species in the measurement region.

During the time period of interest, the satellite was moving from $L=8$ to $L=4$. At $L=4$, the satellite is in the radiation belts. Because this instrument requires a coincidence for a measurement, it is less susceptible to background from penetrating particles than an instrument relying on singles rates. However, when the background rate is high enough, false coincidences are observed. These can appear with equal probability at any time-of-flight. Because the real ions occur as peaks in time-of-flight for a particular energy, they can be easily distinguished from the background if their count rate is sufficiently high.

To illustrate the effect of background, Fig. 1a shows mass spectra from the instrument for the top four energy steps of the mass spectrum product. These correspond to energies $15-40 \mathrm{keV} / \mathrm{e}, 5.8-15 \mathrm{keV} / \mathrm{e}, 2.2-5.8 \mathrm{keV} / \mathrm{e}$ and $1.1-2.2 \mathrm{keV} / \mathrm{e}$. Note that the mass spectrum has much wider energy bands (by a factor of four) than the 3D distribution functions which are used to determine the energy spectra. Because the energy steps are being combined, the mass resolution appears worse than it is. The time period shown is when the spacecraft is at $L=5.75$ during an inbound pass on February 18, 1998 . The background level is indicated with a solid line. The peaks for $\mathrm{H}^{+}, \mathrm{He}^{+}$and $\mathrm{O}^{+}$are clearly visible and distinguishable from background. At the lowest energy, the $\mathrm{O}^{+}$peak is still a factor of four above background. Figure $1 \mathrm{~b}$ shows the mass spectra for a later time period, when the satellite is at $L=4.25 \mathrm{Re}$, and has entered the outer radiation belt, where penetrating energetic electrons cause background in the instrument. At high energies, the peaks for the individual ions are still clearly visible. At lower energies, the proton peak is still significantly above background, but the other species are background dominated. Here we will concentrate on observations of $\mathrm{H}^{+}$and $\mathrm{O}^{+}$, the dominant species, limiting the $\mathrm{O}^{+}$data to where it is well above background.

\section{Observations}

On February 17-18, 1998, a geomagnetic storm with a minimum Dst of -103 occurred. The top four panels of Fig. 2 show the solar wind proton density, $N$, and speed, $V$, the interplanetary magnetic field magnitude, $B$, and clock angle from the Wind spacecraft. The clock angle is measured from the $Z$ axis in the $Y-Z$ gsm plane. The time scale has been shifted by one hour to allow for features observed at the Wind location to reach the Earth. From hour 12 to hour 28, the IMF had a southward component, rotating $180^{\circ}$ from $+Y$ GSM, 
a)
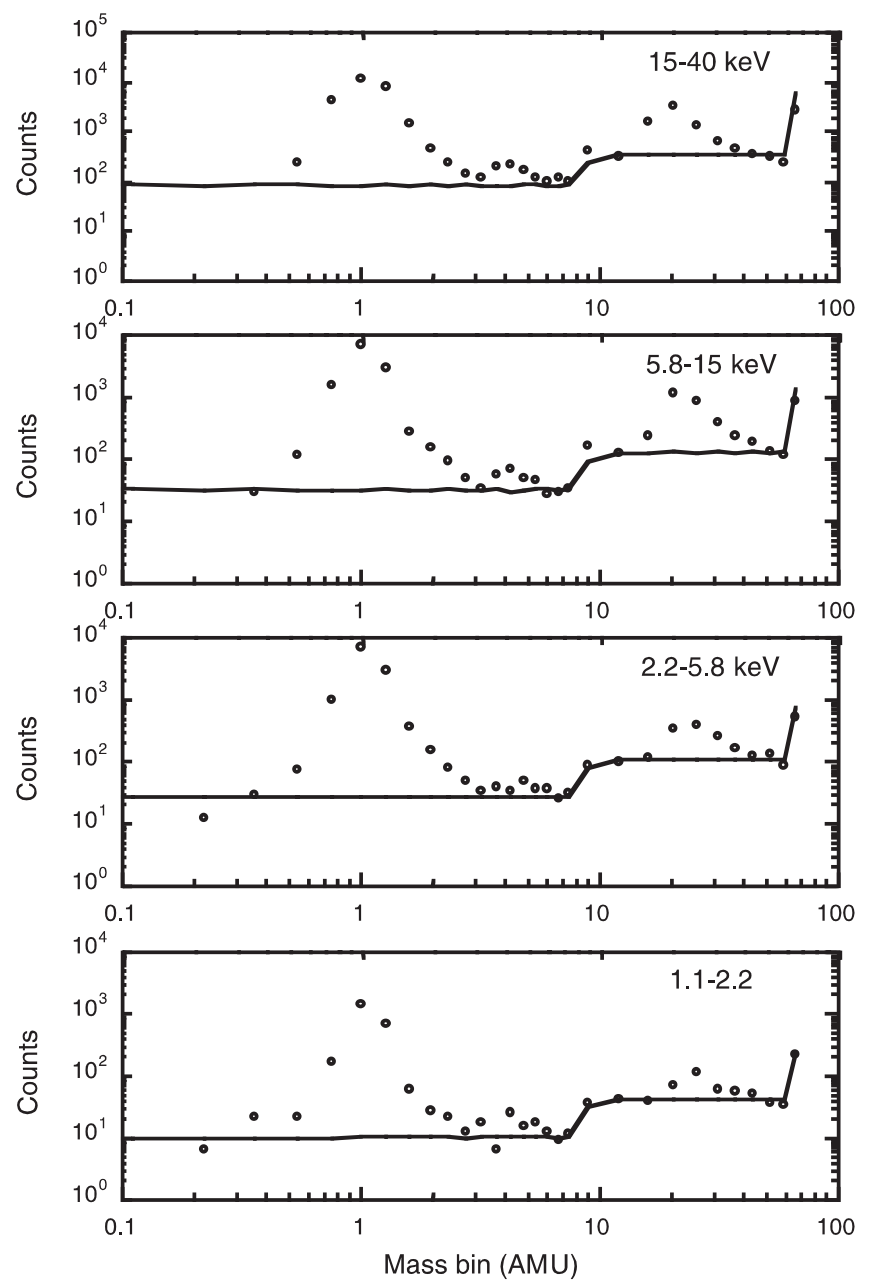

b)
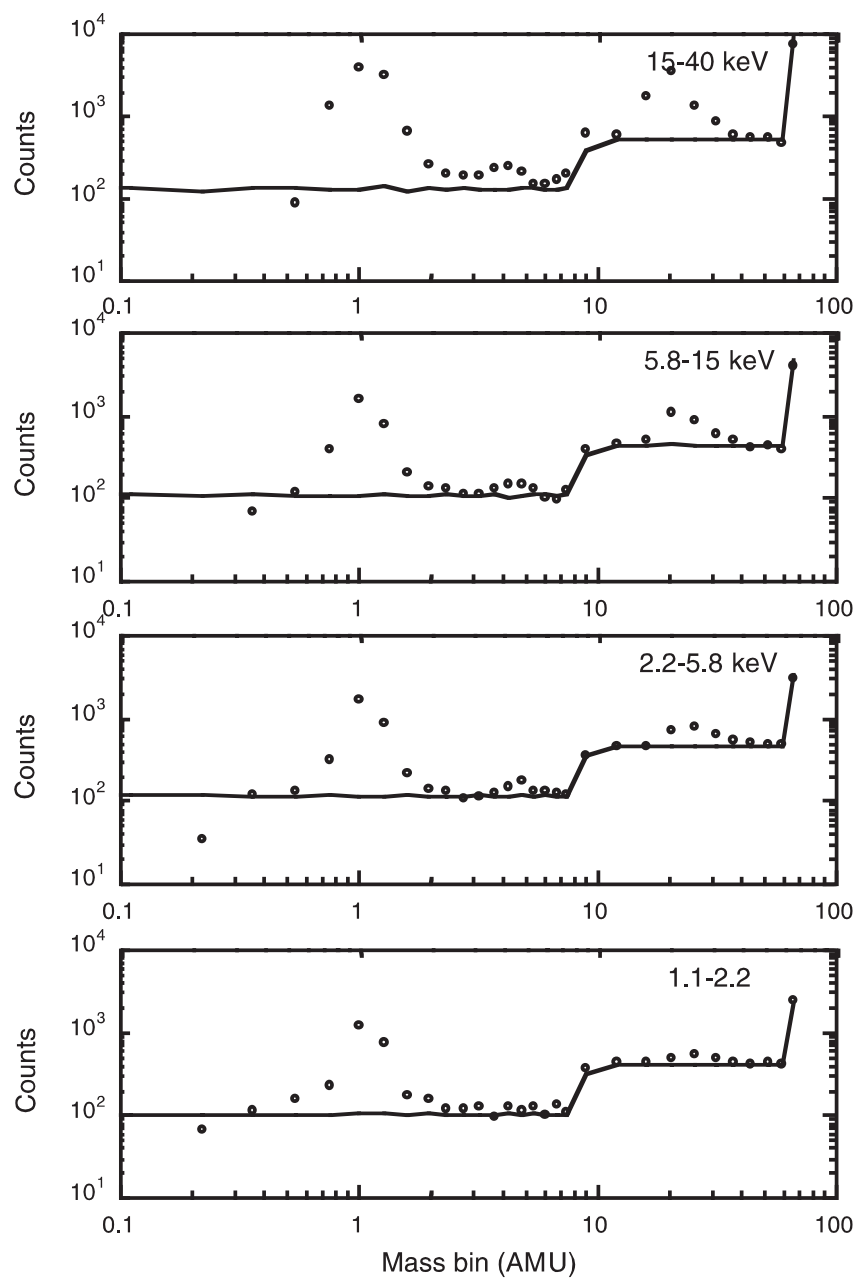

Fig. 1. a Mass distributions at $L=5.75$ for four energy ranges. The solid line gives the background level. The jump in background observed at 9 AMU is due to a discontinuity in the mass algorithm. b Mass distributions at $L=4.25$ in the same format as a

southward, around to $-Y$ GSM. For the next $8 \mathrm{~h}$, the IMF remained in the $-Y$ direction with a small $Z$ component, sometimes north, sometimes south. Panels 5 and 6 show the $D s t$ and $K p$ profiles, respectively. The magnetosphere clearly responded to the southward IMF with a large drop in Dst and an increase in $K p$. The peak of the main phase of the storm occurred at around 00 UT on February 18. Panels 7 and 8 show the $L$-value and magnetic local time of the Equator-S spacecraft. The portion of the Equator-S orbit of interest is given by the heavy line. During this time, Equator-S was moving inbound from $L=8$ to $L=4$ in the pre-noon local time sector. This is the optimum sector for observing the drift effects on the energy spectra.

Figure 3 shows energy time spectrograms of $\mathrm{H}^{+}$and $\mathrm{O}^{+}$from 8:00-11:30, when the spacecraft is moving from $L=8.3$ to $L=4.0$. As the spacecraft moves inbound, a minimum is observed below about $10 \mathrm{keV}$. The width in energy of the minimum increases as the spacecraft moves inbound. A second minimum below about $4 \mathrm{keV}$ is also evident in the protons. The large flux observed below $2 \mathrm{keV}$ in $\mathrm{O}^{+}$after $10: 45$ is due to the penetrating electron background. This masks whether the second minimum is also observed in $\mathrm{O}^{+}$. To show the features more quantitatively, Fig. 4 gives the omnidirectional distribution function, $f$, as a function of energy for $\mathrm{H}^{+}$and $\mathrm{O}^{+}$for one-minute time periods corresponding to $L=5.75,4.75$, and for $\mathrm{H}^{+}$at $L=4.25$. Note that because $f$ is a function in velocity, and not in energy, $\mathrm{O}^{+}$has a higher value in $f$ although its flux is lower. At $L=5.75$, both $\mathrm{H}^{+}$and $\mathrm{O}^{+}$have two minima at about $2 \mathrm{keV}$ and $10 \mathrm{keV}$. At $L=4.75$, the lower energy minimum is just a slight inflection in the curve, and there are now two high energy minima for $\mathrm{H}^{+}$, at 10 and $20 \mathrm{keV}$. The same approximate features are observed at $L=4.25$. Finally, Fig. 5a-c show pitch angle distributions corresponding to $L=5.5-6.0$, and $L=4.5-5.0$ for $\mathrm{H}^{+}$and $\mathrm{O}^{+}$and $L=4.0-4.5$ for $\mathrm{H}^{+}$. The longer time average was used to increase statistics. At $L=5.5-6.0$, the high energy distributions are peaked at $90^{\circ}$ pitch angle. At $10.7 \mathrm{keV}$, the approximate energy of a minimum, a "head-and-shoulders" distribution is observed. Finally, at the low energy minimum, there is a minimum at $90^{\circ}$ pitch angle. The $\mathrm{O}^{+}$ 

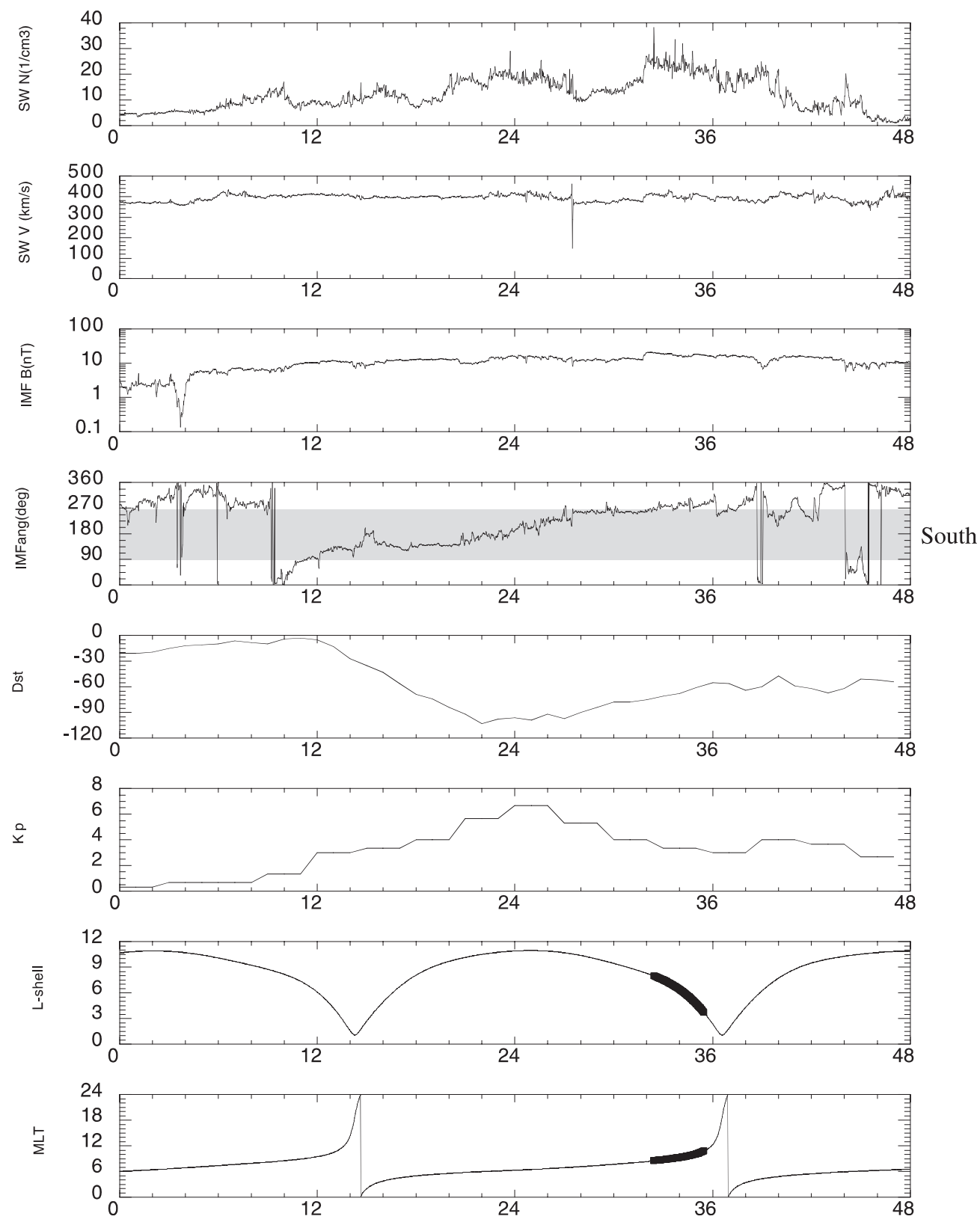

Fig. 2. Solar wind and magnetic field parameters from the MFI and SWE instruments on the Wind spacecraft, $D s t$ and $K p$ indices, and $L$-shell and magnetic local time for the Equator-S spacecraft. The Wind parameters have been shifted in time by one hour

Hours from 0:00 Feb 171998

distributions are similar to those of $\mathrm{H}^{+}$. At lower $L$-values the high and low energy distributions are more isotropic, except for the loss cone. In Fig. 5b, the $\mathrm{H}^{+}$ distribution at $10 \mathrm{keV}$ is strongly peaked at $90^{\circ}$ while the $\mathrm{O}^{+}$is more isotropic.

\section{Modeling}

The energy spectra and pitch angle distributions observed can be used to test models of the magnetospheric convection electric field. To do this we start at the spacecraft location, and model the ion bounce averaged drift paths backwards for $24 \mathrm{~h}$ or until the ion reaches $L=10$. Assuming that charge exchange is the only loss mechanism, we calculate the loss to the distribution function as the ion drifts from the tail to the observation location. Then we assume an initial power law distribution, normalized to the observed spectrum, and calculate the effect that the losses would have on that spectrum. These can then be compared with the observations.

We model the bounce averaged drift paths for two field models: a dipole magnetic field combined with the Volland-Stern electric field, and a dipole magnetic field combined with the Weimer electric field. Both electric fields are time-dependent. The Volland-Stern model is parametrized by $K p$. We use the observed $K p$ values during the storm to change the magnitude of the electric field as a function of time. Since the $K p$ is available for 3$\mathrm{h}$ time periods, we linearly interpolate between the 


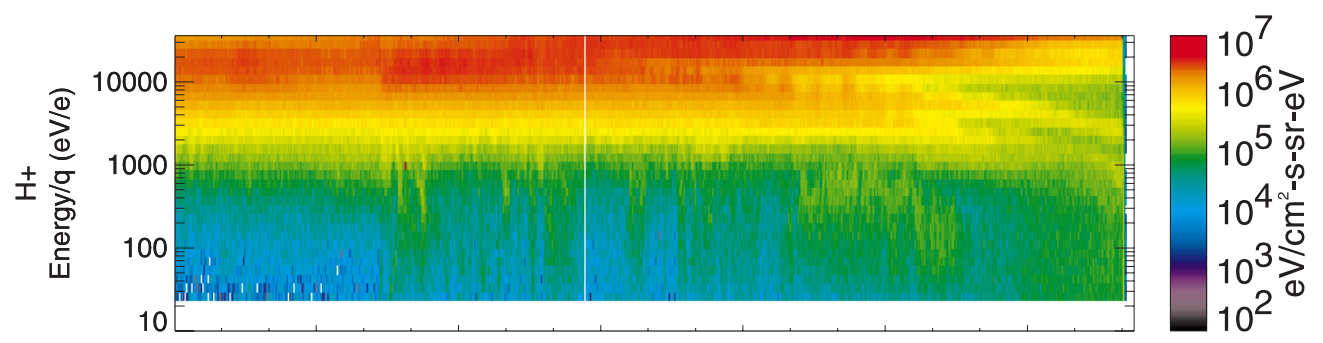

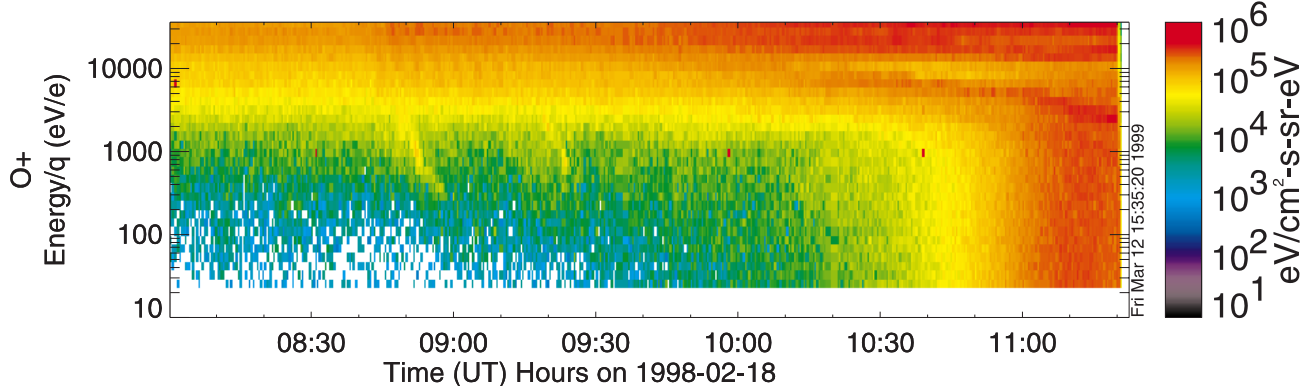

Fig. 3. Energy-time spectrograms for $\mathrm{H}^{+}$and $\mathrm{O}^{+}$for a time period on February 18, 1998, when Equator-S was moving inbound from $L=8.3$ to $L=4.0$ in the pre-noon local time sector different $K p$ levels, which results in a slow time variation. The Weimer electric field is parametrized by the solar wind speed, the IMF direction, and the dipole tilt. We have used values from MFI and SWE instruments on the Wind spacecraft, assuming a travel time of 1-h. In this case the values are updated every $5 \mathrm{~min}$, so the time variation is much faster.

Figure 6 shows the drift paths of the ions which are observed at $L=5.75$, and 9.57 MLT. The paths are shown for six energies from $35.8 \mathrm{keV}$ down to $3.2 \mathrm{keV}$. The distance between the symbols indicates 1 hour of drift time. Figure $6 a-c$ is for the Volland-Stern electric field for three different pitch angles, and Fig. $6 \mathrm{~d}-\mathrm{f}$ for the Weimer electric field for the same pitch angles. One obvious difference is that the Volland-Stern drift paths are much more regular. This is due to both the simple analytical form of the field, and the slower time variation. For drifts in the Volland-Stern electric field, the transition region between eastward and westward drifting paths is between 3 and $5 \mathrm{keV}$. For the Weimer electric field, the transition energy is a little higher, particularly at the smaller pitch angles. At $45^{\circ}$ and $10^{\circ}$,
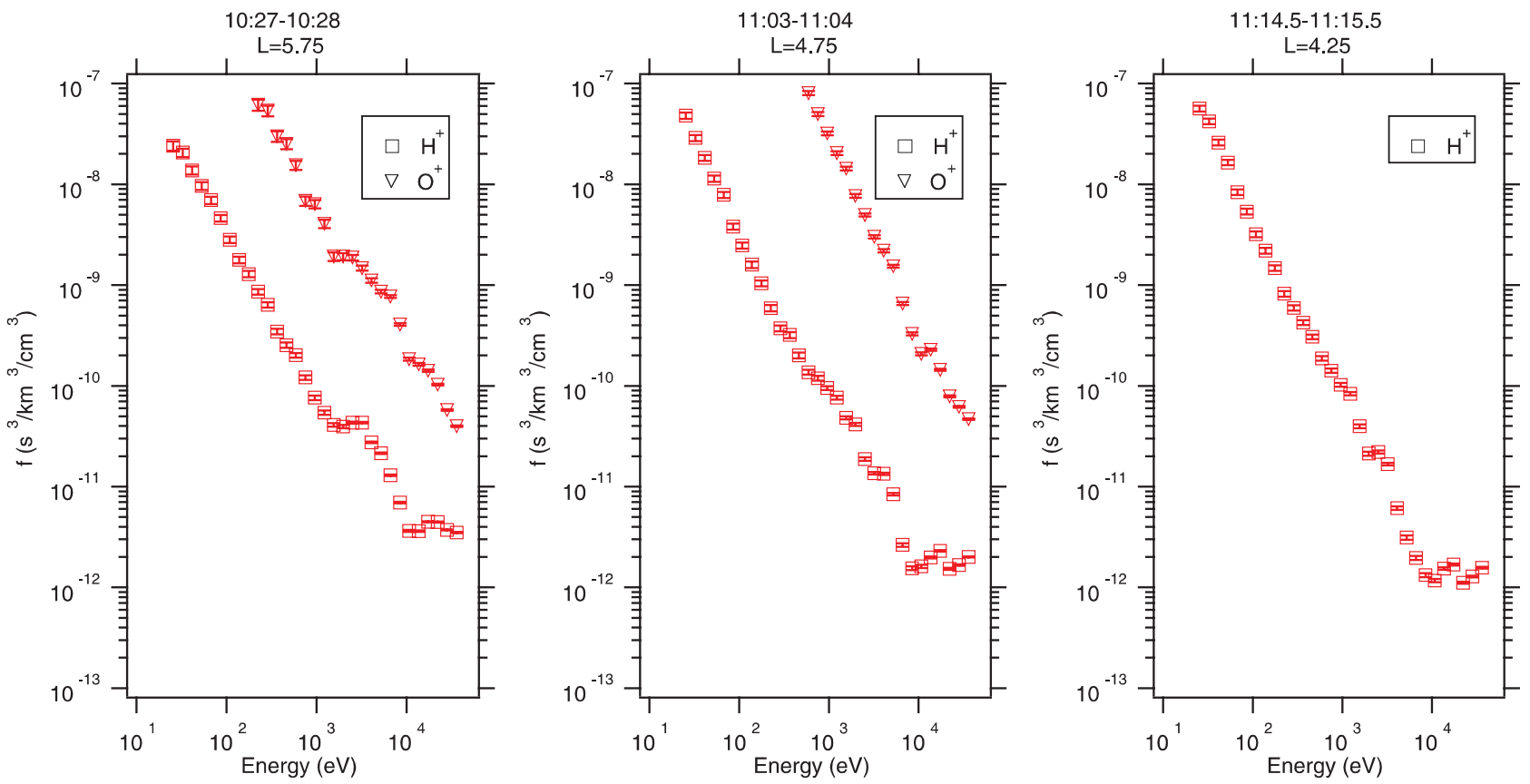

Fig. 4. Ion distribution function versus energy for 1 -min time periods at $L=5.75, L=4.75$, and $L=4.25$. $\mathrm{O}^{+}$is not shown at $L=4.25$ because the low energy range is contaminated by background at this time 

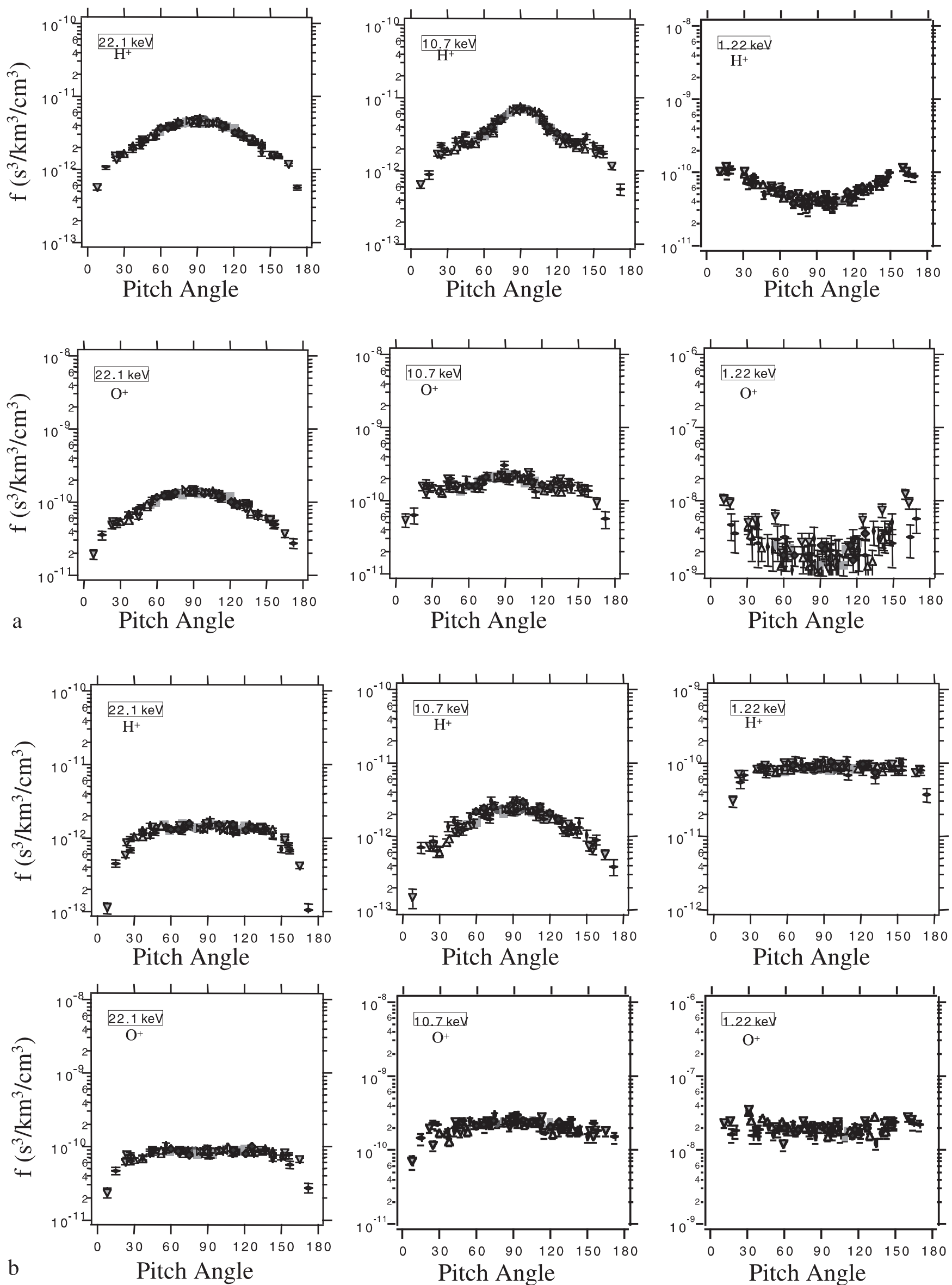

Pitch Angle 


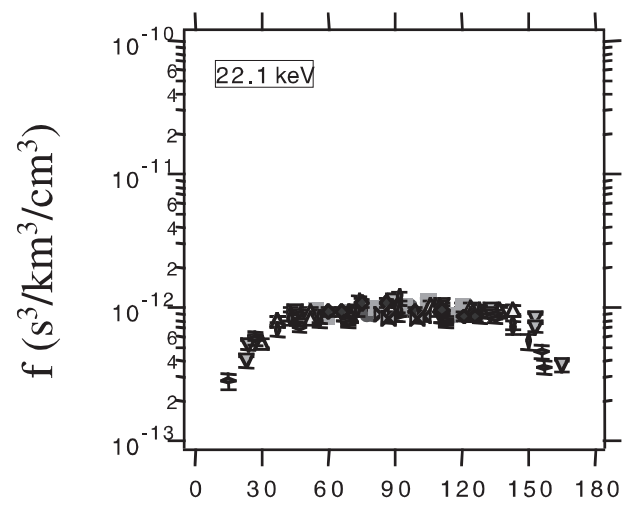

c

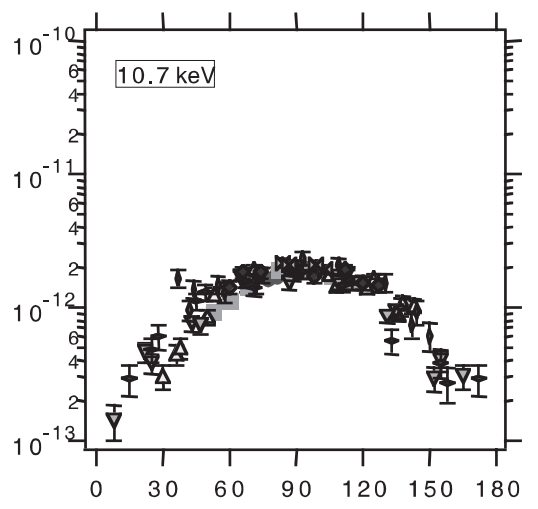

Pitch Angle

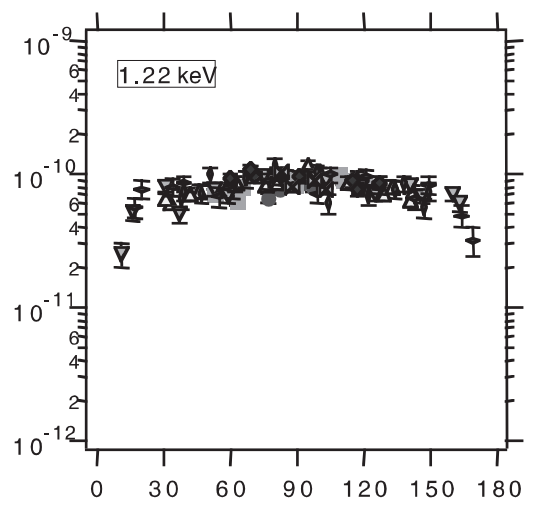

Pitch Angle
Fig. 5. a Pitch angle distributions at three different energies for $\mathrm{H}^{+}$ and $\mathrm{O}^{+}$at $L=5.5-6.0$. The different symbols represent different look directions with respect to the spin axis in the instrument. b Pitch angle distributions at three different energies for $\mathrm{H}^{+}$and $\mathrm{O}^{+}$at $L=4.5$ 5.0. The different symbols represent different look directions with respect to the spin axis in the instrument. $\mathbf{c}$ Pitch angle distributions at three different energies for $\mathrm{H}^{+}$at $L=4.0-4.5$. The different symbols represent different look directions with respect to the spin axis in the instrument a) Volland-Stern Field, $89^{\circ}$ Pitch Angle

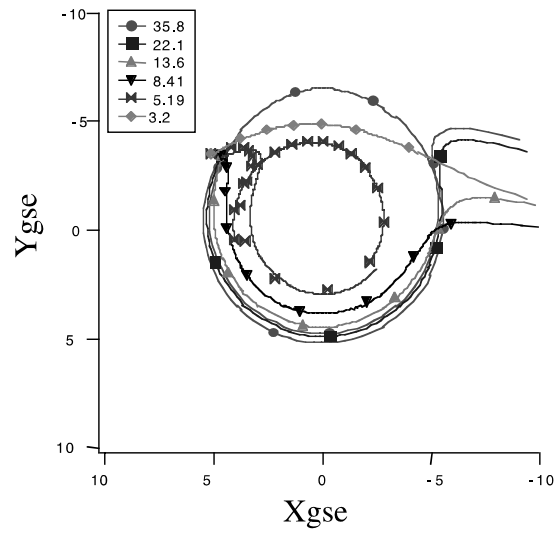

d) Weimer Field, $89^{\circ}$ Pitch Angle

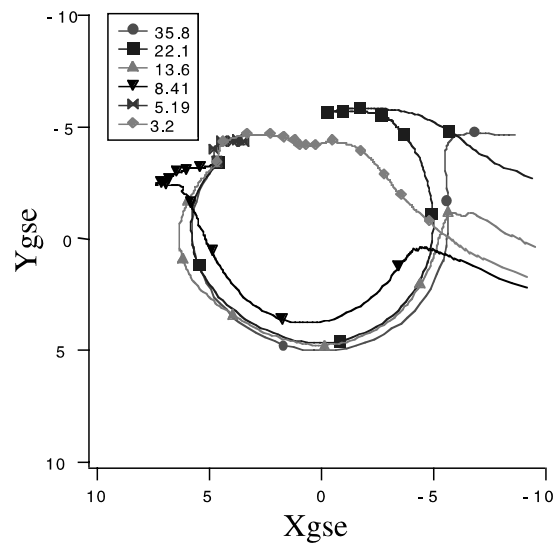

b) Volland-Stern Field, $45^{\circ}$ Pitch Angle

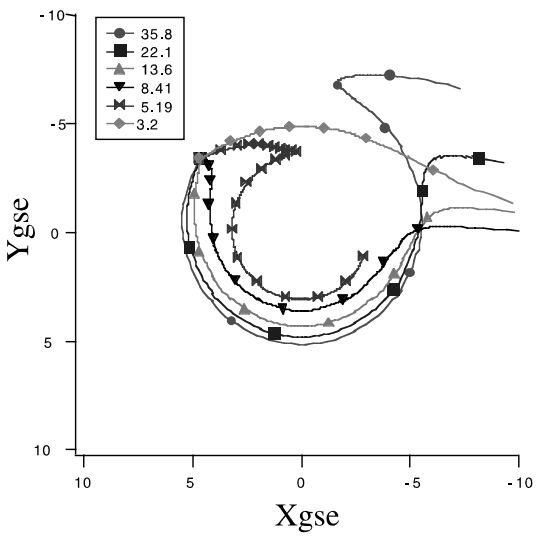

e) Weimer Field, 45 Pitch Angle

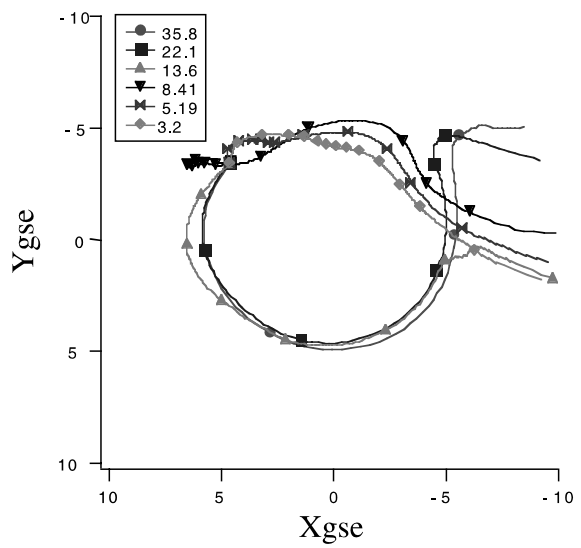

c) Volland-Stern Field, $10^{\circ}$ Pitch Angle

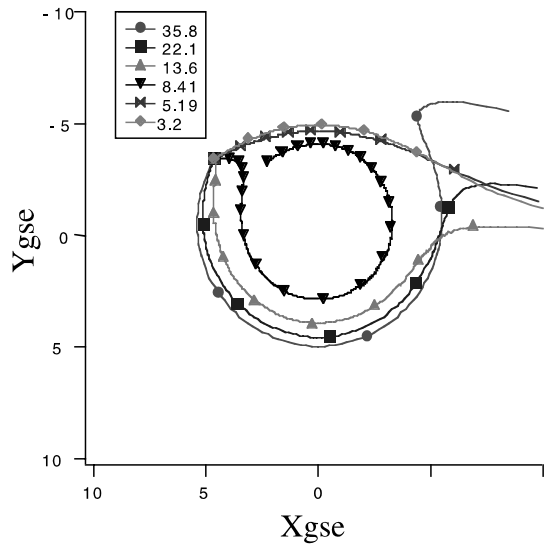

f) Weimer Field, $10^{\circ}$ Pitch Angle

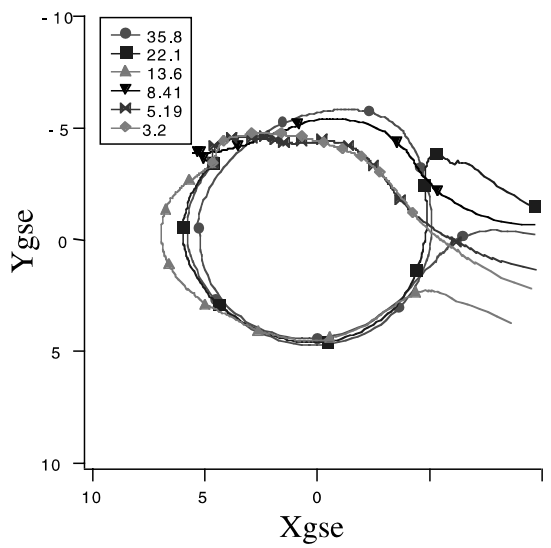

Fig. 6a-f. Bounce averaged drift paths for ions which arrive at $L=5.75,9.57$ MLT with 6 different energies. The paths were calculated using a dipole magnetic field and either a Volland-Stern electric field $(\mathbf{a}-\mathbf{c})$ or a Weimer electric field $(\mathbf{d}-\mathbf{f})$. The legend gives the ion energy in keV

the transition energy is between 8 and $13 \mathrm{keV}$. Another difference is that the eastward drift in the Weimer field is significantly slower than in the Volland-Stern field (compare the $3.2 \mathrm{keV}$ path in all cases). Finally, the westward Volland-Stern drift paths tend to go closer to the Earth than the Weimer paths. For both fields, the transition energy is higher at lower pitch angles. This can lead to the "head and shoulders" type pitch angle 
a) Volland-Stern Field, $89^{\circ}$ Pitch Angle

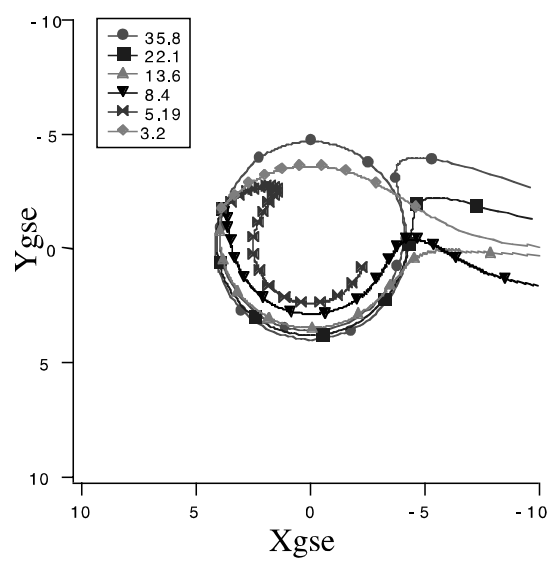

d) Weimer Field, 89० Pitch Angle

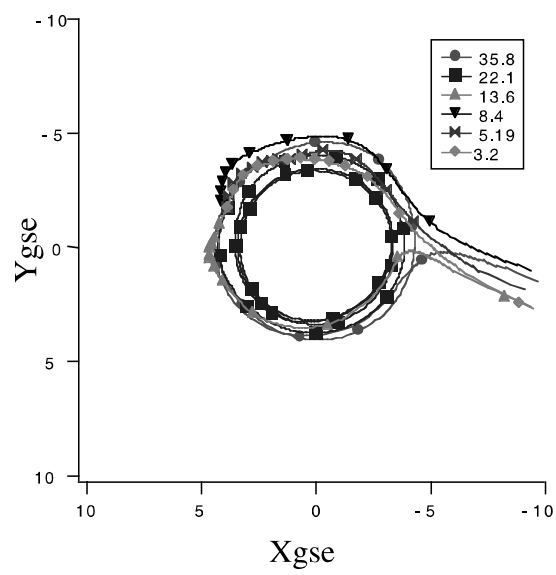

b) Volland-Stern Field, 45 ${ }^{\circ}$ Pitch Angle

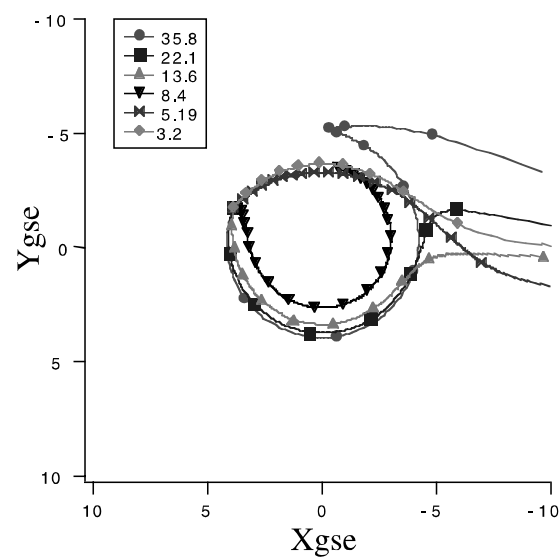

e) Weimer Field, $45^{\circ}$ Pitch Angle

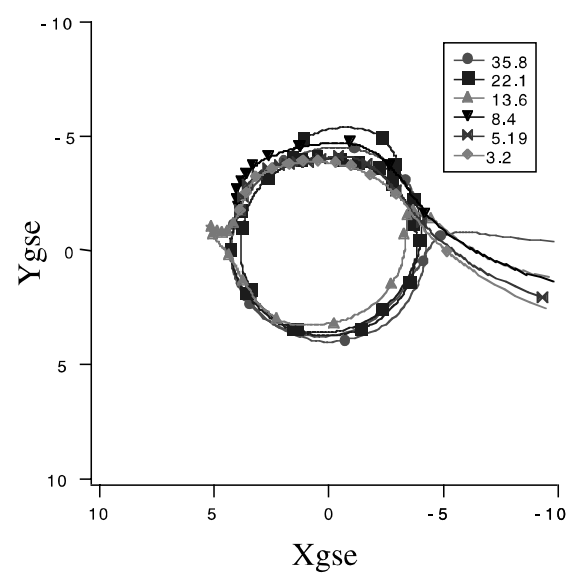

c) Volland-Stern Field, $10^{\circ}$ Pitch Angle

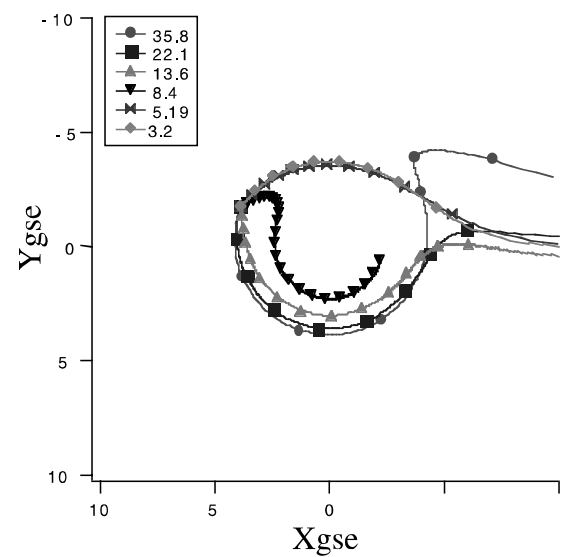

f) Weimer Field, $10^{\circ}$ Pitch Angle

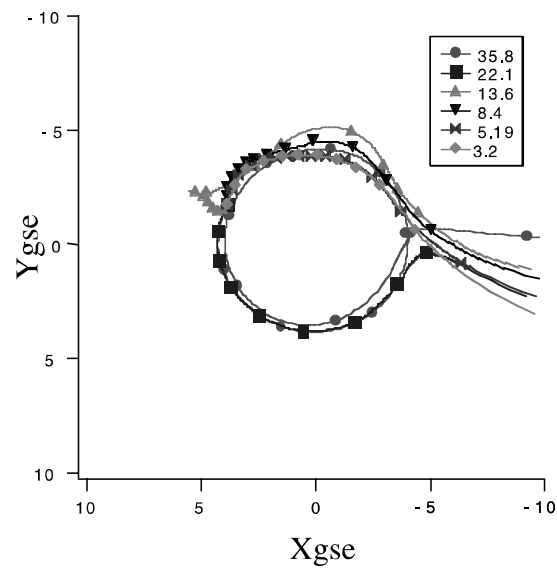

Fig. 7a-f. Bounce averaged drift paths for ions which arrive at $L=4.25,10.41$ MLT with 6 different energies. The paths were calculated using a dipole magnetic field and either a Volland-Stern electric field $(\mathbf{a}-\mathbf{c})$ or a Weimer electric field $(\mathbf{d}-\mathbf{f})$. The legend gives the ion energy in keV

distribution observed at $10.7 \mathrm{keV}$ in Fig. 5a if the energy corresponds to the range where the low pitch angle ions drift eastward while the high pitch angle ions drift westward, like the drift path of an $8.4 \mathrm{keV}$ ion in the Weimer model.

Figure 7 compares the drift paths at $L=4.25$ and MLT $=10.41$. The difference between the transition energies for this $L$-value and local time is larger: the transition energy in the Volland-Stern electric field is between 3 and $5 \mathrm{keV}$, for the $89^{\circ}$ pitch angle ions, and between 5 and $8 \mathrm{keV}$ at lower pitch angles, while for the Weimer field it is between 8 and 13.6 for $89^{\circ}$ and $45^{\circ}$ pitch angle, and greater than 13.6 for the ions at $10^{\circ}$. Again, the eastward drift in the Weimer field is slower than for the Volland-Stern field.

Figure 8 shows the percent of the distribution function that will remain after the ions have drifted in from $L=10$. The percent remaining is shown as a function of energy for the two models and for three different pitch angles. We have used the charge exchange cross sections given by Smith and Bewtra (1978) with the neutral hydrogen geocorona model of Rairden et al. (1986). $\mathrm{H}^{+}$and $\mathrm{O}^{+}$results at $L=5.75$ are shown, and $\mathrm{H}^{+}$only at $L=4.25$. Using the Volland-Stern electric field gives one deep minimum, which corresponds to the region around the transition energy. Interestingly, the predicted distributions using the Weimer electric field have multiple minima. A broad dip is observed, with its minimum from 3-5 keV (depending on pitch angle). An examination of the drift trajectories in Figs. 6 and 7 shows that this minimum results from the slow eastward drift paths. The Weimer field then produces second and third minima corresponding to the transition energy and above. The highest energy minima result from particular ion energies which make multiple transits around the Earth before reaching the spacecraft location. These types of trajectories would be very sensitive to the time dependence of the field. $\mathrm{O}^{+}$, in general loses less, due to its smaller cross section for charge exchange at these energies. This is consistent with our observation in Fig. $5 \mathrm{~b}$ that the the $\mathrm{O}^{+}$is more isotropic at $10.7 \mathrm{keV}$, the energy close to the minimum, than $\mathrm{H}^{+}$, and is also consistent with the smaller minima observed in the spectra in Fig. 4.

Figure 9 shows a comparison of the measured distribution functions as a function of energy for $\mathrm{H}^{+}$ and $\mathrm{O}^{+}$with the predicted spectra using the two electric fields. The predicted spectra are determined by assuming 

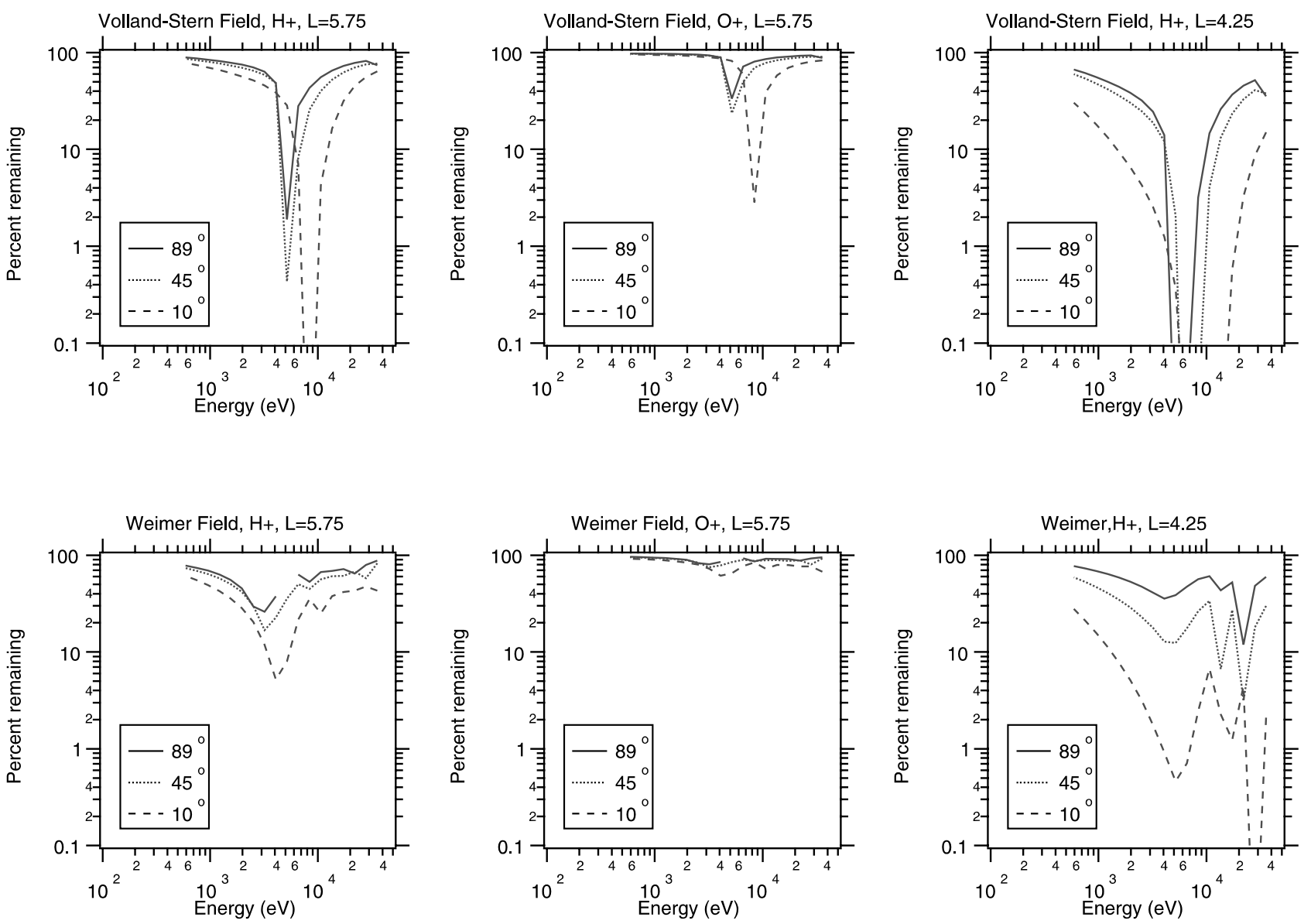

Fig. 8. The percent of the distribution that remains, as a function of energy, after the ions have drifted from the tail to the observation location. The top panels assume a convection in a Volland-Stern electric field, and the bottom panels assume convection in a Weimer electric field. Charge exchange with the neutral hydrogen geocorona is the only loss mechanism assumed an initial power law spectrum (a straight line in this log$\log$ representation). Power-law spectra were consistently observed by Kistler et al. (1989) on the night-side during storms, over the energy range $1-50 \mathrm{keV}$. We then apply the losses as shown in Fig. 8 to these spectra. These comparisons should therefore be used to determine how well the model represents the features in the spectra, but not the overall normalization or slope of the spectra. It is also possible that features result from changes in the input spectrum with time, spatial differences in the spectrum from the different tail regions that contribute to the spectra, or from deviations from a power law that were present in the input tail spectrum. These types of effects would not appear in our "model" spectra.

For both input fields, we have compared the omnidirectional energy spectra with the losses calculated for ions with pitch angles of $10^{\circ}, 45^{\circ}$, and $89^{\circ}$. Using the Volland-Stern electric field gives too large a loss. When the Weimer electric field is used, the magnitude of the losses is much closer to that observed. The smaller $\mathrm{O}^{+}$ cross section for charge exchange at these energies leads to less pronounced minima in the spectra, as observed. In addition, the Weimer electric field is better for predicting the complexity of the spectra observed. It predicts a low energy broad minimum, which is observed at about $2 \mathrm{keV}$, and multiple high energy minima, as are observed. However, the energies of the minima predicted do not quantitatively agree with the observed energies.

As a final test, we have compared the pitch angle distributions observed with those predicted by the model. As noted previously, the head-and-shoulders distributions result from the low pitch angle ions drifting eastward while the high pitch angle ions drift westward. Since our model does not reproduce the energy of this transition quantitatively, it is difficult to do a quantitative pitch angle comparison in this region. Therefore, we have restricted our comparisons to energies slightly away from the predicted and observed minima. Figure 10 compares the observed $\mathrm{H}^{+}$and $\mathrm{O}^{+}$pitch angle distributions at $L=4.5-5.0$ with the predicted distributions using the Volland-Stern electric field (solid line) and the Weimer electric field (dotted line) for four energies. To generate our predicted distributions we have assumed an isotropic distribution injected on the nightside. Both models reproduce the observed distributions very well at high energies ( $28 \mathrm{keV}$ and $13.6 \mathrm{keV})$ and at low energies $(1.2 \mathrm{keV})$. At $8.4 \mathrm{keV}$, which is the 

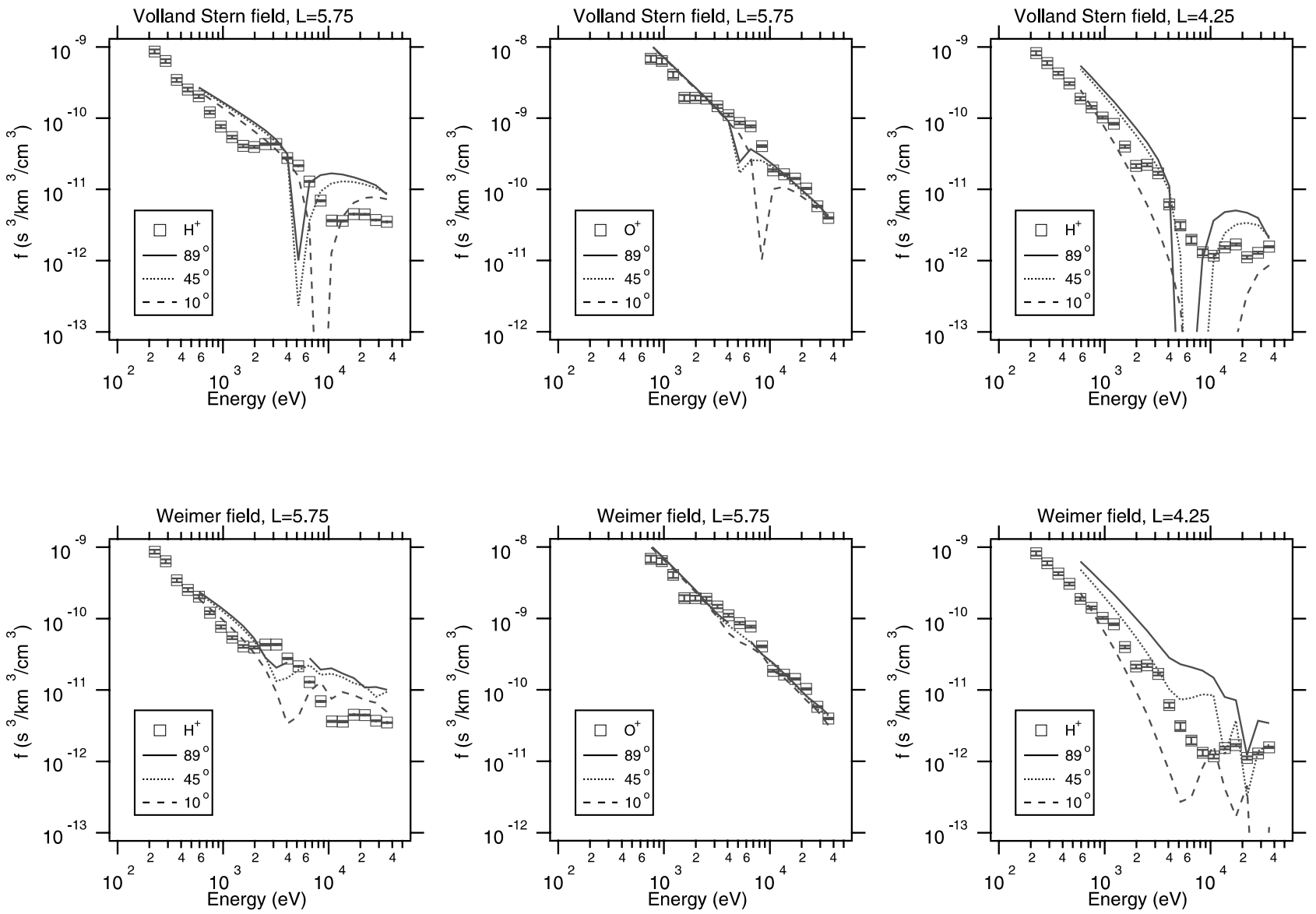

Fig. 9. Comparisons of observed spectra and modeled spectra using the Volland-Stern electric field (top panels) and the Weimer electric field (bottom panels) assuming charge exchange is the only loss mechanism. The symbols show the data, and the lines give the model results
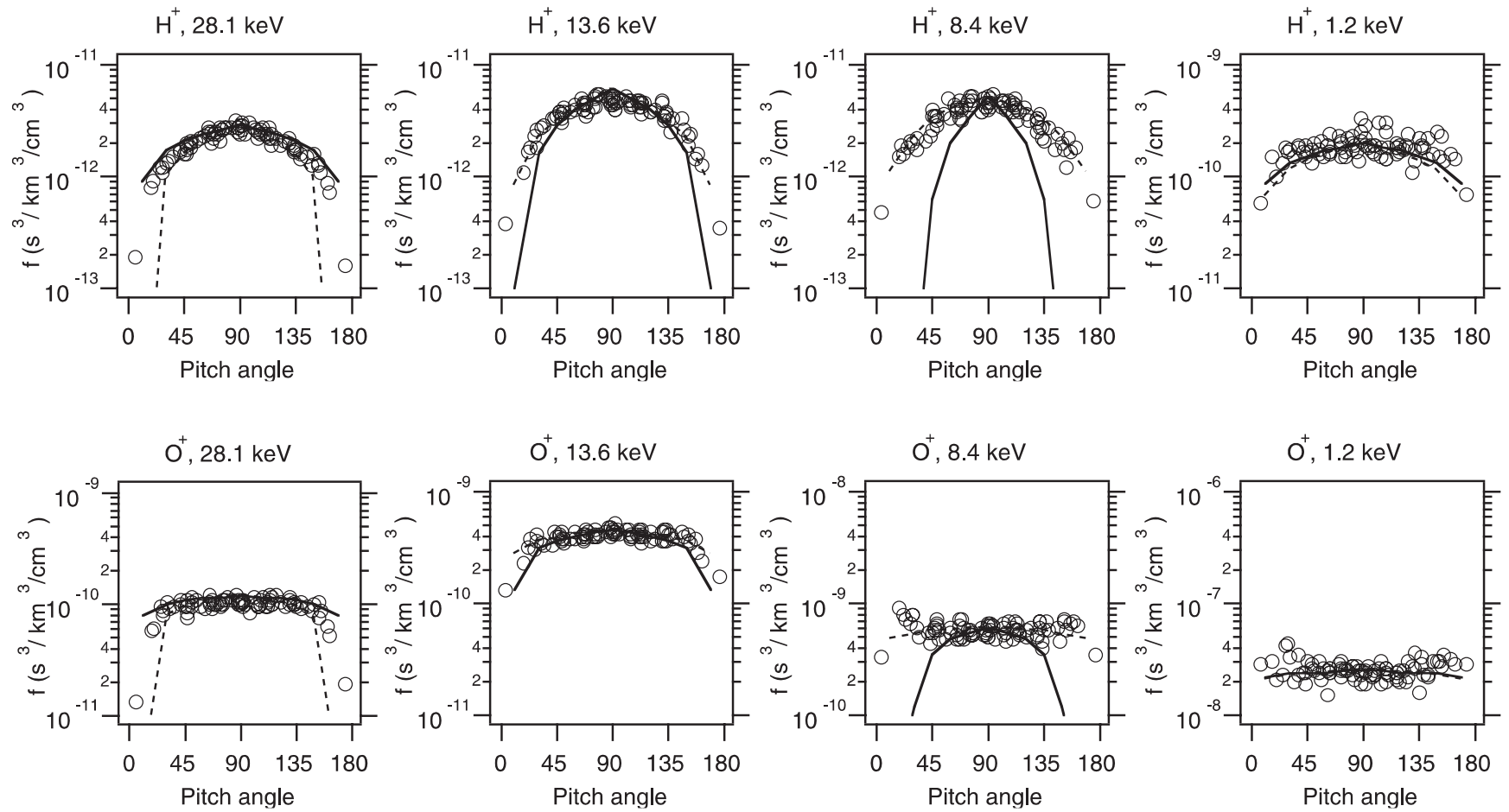

Fig. 10. Comparisons of observed and modeled pitch angle distributions using the Volland-Stern electric field (solid line) and the Weimer electric field (dashed line) assuming an initial isotropic distribution and charge exchange as the only loss mechanism 
closest to the predicted minimum, the Volland-Stern model predicts losses which are too large, resulting in a more highly peaked distribution than is observed. The observed $\mathrm{O}^{+}$distributions at $8.4 \mathrm{keV}$ and to a lesser extent at $1.2 \mathrm{keV}$ increase at pitch angles away from $90^{\circ}$. This is not reproducted by our models, and indicates that the injected $\mathrm{O}^{+}$spectrum on the nightside at these energies was field-aligned, not isotropic.

\section{Conclusions}

We have used observations of the ion energy spectra using the Equator-S satellite to test the validity of two models of the storm-time convection electric field. This is the first time that the Weimer model has been tested for its validity in the equatorial plane using drift trajectories. We find that assuming convection in a Volland-Stern electric field with charge exchange as the only loss mechanism results in predicted losses that are larger than observed. This was evident in both the predicted energy spectra and the predicted pitch angle distributions. This is in agreement with the findings of Jordanova et al. (1999) from comparisons with POLAR data. Chen et al. (1998) in comparing simulating pitch angle distributions with CRRES data for higher energy ions $(>50 \mathrm{keV} / \mathrm{e})$ found the opposite result. Assuming convection in the Weimer field gives significantly better agreement with the magnitude of the losses observed. From comparisons of the drift paths, this is primarily because the Volland-Stern trajectories in the transition region go much closer to the Earth. The differences in charge exchange cross sections of $\mathrm{H}^{+}$and $\mathrm{O}^{+}$at these energies is observed in the spectra and pitch angle distributions, and is accurately reproduced by the models, confirming our assumption that charge exchange is the most significant loss process at these $L$ values and energies. Thus the quantitative differences more likely lie in the magnetic and electric field models used. The Weimer field accurately reproduces the complex spectra with multiple minima that are observed during this storm. However, it does not quantitatively predict the energies of the minima.

We have shown that using a more realistic electric field significantly improves the agreement with the observed spectra. In future work, we will also include a more realistic magnetic field, both in calculating the trajectories, and in mapping the Weimer electric potential up the field line to test if this will finally give quantitative agreement between the model spectra and the observations.
Acknowledgements. We are grateful to the many engineers and scientists from UNH, MPE, CESR, MPAe, IFSI, IRF, UCB and UW who made the development of the ESIC instrument possible. Development of the ESIC instrument in the US and this work were supported by NASA grants NAG5-4408 and NAG5-6925. The modeling was funded in part by NAG5-4695. The Equator-S mission was financially supported via grant $50 \mathrm{OC} 94024$ by the German Space Agency, DARA (now DLR). We thank R. Lepping and K. Ogilvie for use of the Wind MFI and SWE key parameter data used in this study.

The Editor-in-Chief thanks M. C. Fok and M. W. Chen for their help in evaluating this paper.

\section{References}

Chen, M. W., J. L. Roeder, J. F. Fennel, L. R. Lyons, and M. Schulz, Simulations of ring current proton pitch angle distributions, J. Geophys. Res., 103, 165, 1998.

Fok, M.-C., T. E. Moore, and M. E. Greenspan, Ring current development during storm main phase, J. Geophys. Res., 101, $15311,1996$.

Jordanova, V. K., C. J. Farrugia, J. M. Quinn, R. B. Torbert, J. E. Borovsky, R. B. Sheldon, and W. K. Peterson, Simulations of offequatorial ring current ion spectra measured by Polar for a moderate storm at solar minimum, J. Geophys. Res., 104, 429, 1999.

Kistler, L. M. et al., Energy spectra of the major ion species in the ring current during geomagnetic storms, J. Geophys. Res., 94, 3579, 1989.

Maynard, N. C., and A. J. Chen, Isolated cold plasma regions: observations and their relation to possible production mechanisms, J. Geophys. Res., 80, 1009, 1975.

Möbius, E., et al., The 3-D plasma distribution function analyzers with time-of-flight mass discrimination for CLUSTER, FAST, and Equator-S, measurement techniques for space plasmas: J. Borovsky, R. Pfaff, D. Young, Eds., American Geophysical Union, Washington, DC, 2431998.

Rairden, R. L., L. A. Frank, and J. D. Craven, Geocoronal imaging with Dynamics Explorer, J. Geophys. Res., 91, 13 613, 1986.

Rème, H., et al., The Cluster ion spectrometry experiment, Space Sci. Rev., 79, 303-350, 1997.

Smith, P. H., and N. K. Bewtra, Charge exchange lifetimes for ring current ions, Space Sci. Rev., 22, 301, 1978.

Stern, D. P., The motion of a proton in the equatorial magnetosphere, J. Geophys. Res., 80, 595, 1975.

Volland, H., A semiempirical model of large-scale magnetospheric electric fields, J. Geophys. Res., 78, 171, 1973.

Weimer, D. R., Models of high-latitude electric potentials derived with a least error fit of spherical harmonic coefficients, $J$. Geophys. Res., 100, 19 595, 1995.

Weimer, D. R., A flexible, IMF dependent model of high-latitude electric potentials having "space weather" applications, Geophys. Res. Lett., 23, 2549, 1996.

Wygant, J., D. Rowland, H. J. Singer, M. Temerin, F. Mozer, and M. K. Hudson, Experimental evidence on the role of the large spatial scale electric field in creating the ring current, $J$. Geophys. Res., 103, 29 527, 1998. 